\title{
Representaciones utilizadas por los estudiantes para resolución de problemas matemáticos del mundo físico y social
}

\author{
Representations used by students to solve mathematical problems \\ in the physical and social world
}

\author{
Fabiola Elena Guerrero Salazar fabiolaguerrero584@gmail.com \\ Código ORCID: 0000-0002-6908-0139 \\ Universidad de Carabobo, Venezuela
}

\author{
Kelly Dayhana Bravo Ferreira \\ bravo.kelly753@hotmail.com \\ Código ORCID: 0000-0003-2286-5454 \\ Universidad de Carabobo, Venezuela
}

Recibido abril 2020 | Arbitrado mayo 2020 | Publicado junio 2020

\begin{abstract}
Resumen
Palabras clave:

Representaciones; problemas matemáticos; mundo físico y social

La presente investigación tuvo como propósito determinar las representaciones utilizadas por los estudiantes de primer año cuando resuelven problemas matemáticos del mundo físico y social de la Unidad Educativa "El Molino". El estudio se fundamentó en los organizadores curriculares desarrollados por Rico (1997), los cuales son necesarios para que los estudiantes integren con sentido los nuevos conocimientos y se apropien de ellos. El paradigma fue cualitativo, con un enfoque interpretativo, basado en estudio de casos, donde se aplicó la entrevista en profundidad y cuestionario de problemas matemáticos, específicamente a treinta (30) estudiantes que sirvieron de informantes clave. Concluyendo, es necesario abordar los contenidos matemáticos a través de la resolución de problemas reales, que preparen a los estudiantes para conocer su entorno; además, formar a los estudiantes para el estudio del álgebra, pues el sistema más utilizado por los mismos fue el verbal y numérico, el cual contribuyen en desarrollar un pensamiento crítico.
\end{abstract}

\section{Abstract}

Keywords:

Representations; mathematical problems; physical and social world
The purpose of this research was to determine the representations used by firstyear students when solving mathematical problems in the physical and social world of the El Molino Educational Unit. The study was based on the curricular organizers developed by Rico (1997), which are necessary for students to meaningfully integrate new knowledge and appropriate it. The paradigm was qualitative, with an interpretive approach, based on case studies, where the indepth interview and questionnaire of mathematical problems were applied, specifically to thirty (30) students who served as key informants. In conclusion, it is necessary to approach the mathematical contents through the resolution of real problems, which prepare students to know their environment; also, train students to study algebra, since the most used system by them was verbal and numerical, which contribute to developing critical thinking. 


\section{INTRODUCCIÓN}

Los cambios en la sociedad actual a través de la globalización han ocasionado la necesidad de que sus habitantes tomen decisiones acertadas y den respuestas a los problemas de su entorno; se cree que la Matemática es la base para formar en las personas, el pensamiento crítico y reflexivo que los ayude a vivir en sociedad y que les brinde las herramientas necesarias para comprender su entono social y para poder modificarlo si es preciso. Por tal motivo, se considera propicio que la educación que reciban los estudiantes, sea contextualizada, adaptada a sus necesidades, y que incorpore actividades de resolución de problemas que le permitan acostumbrarse $y$ adquirir habilidades para esta actividad.

En las instituciones escolares, la matemática junto con otras áreas forma parte del curriculum escolar y es obligatoria para todos los niveles tanto en Venezuela como en el mundo, siendo considerada al igual que el lenguaje como un área fundamental, por su importancia en el desarrollo del análisis y la comunicación de las ideas; además de que permite desarrollar en el estudiante aspectos como la creatividad, la capacidad de análisis y síntesis y el razonamiento crítico, entre otros. (Luengo González, R., Blanco Nieto, L., Mendoza García, M., Sánchez Pesquero, C., Márquez Zurita, L. y Casas García, L.M., 1997). Por tal motivo, y a pesar de su alto grado de abstracción, precisión $y$ formalidad, la matemática debe ser enseñada y aprendida de manera dinámica y abierta, que propicie la construcción de su conocimiento, con la realización de actividades concretas, con objetos reales y sobre objetos reales, la propia realidad del educando, y contextualizándola con el mundo físico y social que lo rodea.

Según Rico (1997), la matemática escolar necesita de una pluralidad de significados de manera que los estudiantes puedan integrar con sentido los nuevos conocimientos.

Para estudiar estos significados de manera sistemática se encuentran los organizadores del currículo de matemática. Por lo cual, la fenomenología didáctica, los sistemas de representación, los modelos matemáticos y procesos de modelización, los materiales y recursos, los errores, dificultades y obstáculos, la historia de la matemática y, por último, la resolución de problemas son considerados como organizadores curriculares (Rico, 1997). Los mismos deberían estar presentes en el trabajo matemático, desarrollados en las aulas de matemática, para lograr que los estudiantes adquieran nuevos conocimientos y le asignen significados a los conceptos matemáticos.

Sin embargo, Jaime y Gutiérrez (1990) manifiestan que los estudiantes no logran comprender nuevos conceptos, y en el caso de que lo hagan, sólo son capaces de usarlos con ejemplos idénticos a los resueltos con la ayuda del profesor, además pueden resolver problemas concretos con bastante habilidad, pero cuando les corresponde realizar esos mismos problemas en otros contextos carecen de ideas y no pueden resolverlos, y por último tienden a memorizar las demostraciones y las formas de resolver los ejercicios, para aprobar las evaluaciones; lo que muestra que la manera cómo se ha estado enseñando la matemática no ha ido en conformidad, con el trabajo que requiere la asignatura en las escuelas. 
En vista de esta situación, la forma cómo se enseña y cómo se adquiere el conocimiento matemático ha llevado a reestructuraciones curriculares, en los años sesenta, los procesos de revisión de los fundamentos de la Matemática condujeron a la presentación de la llamada "Matemática Moderna" por parte del grupo Bourbaki, el cual propuso tomar como punto de partida a la lógica formal y la teoría de conjuntos y obtener a partir de allí la estructura axiomática y sistemática de las mismas. Desde ese momento, en las escuelas, la matemática comenzó a enseñarse con un enfoque axiomático y el resto de sus disciplinas matemáticas fueron opacadas por el Álgebra, la cual es considerada como una de las partes de la matemática que influye considerablemente en el aspecto formativo, por la potencia y simplicidad de sus registros formales y sus métodos. (Socas, Camacho y Hernández, 2008) aunque al parecer es vista desde su forma más elemental; representar números con símbolos como ( $\mathrm{a}, \mathrm{b}, \mathrm{x}, \mathrm{y}, \mathrm{z})$, obviando su funcionalidad.

El estudio del Álgebra comprende, lo que para Katz (2007) son las principales ideas de la Matemática, la Generalización y la Abstracción, las cuales ayudan a que los estudiantes desarrollen habilidades para la resolución de problemas, y problemas propios del mundo real y de su cotidianidad, haciendo uso de las reglas de la Aritmética como herramienta para dar solución a los mismos. Sin embargo, este uso de la Aritmética ha ocasionado, a través de los años, que el Álgebra sea considerada una generalización de la Aritmética, siendo un hecho poco provechoso para el proceso de enseñanza y aprendizaje de la matemática, pues ha impedido la adquisición de las habilidades para la resolución de problemas y la degeneración de una secuencia de habilidades algebraicas que preparan al estudiante para el Álgebra superior (Katz, 2007).

A pesar de que el fenómeno de la "Matemática Moderna" cambió la enseñanza de la Matemática dándole una primordial importancia al Álgebra, en el doceavo estudio ICMI, Stacey y Chick (2004) señalan que la misma es el área problemática de la enseñanza de la Matemática porque presenta complicaciones de pertinencia y equidad. De equidad por la masificación de los sistemas escolares donde se requieren grandes destrezas de los docentes para aprovechar las ventajas de los estudiantes más aplicados $\mathrm{y}$ al mismo tiempo lograr buenos resultados en los estudiantes con diferentes capacidades e intereses. $Y$ de pertinencia porque en las escuelas el Álgebra simbólica es presentada como una simple manipulación de letras, lo ha ocasionado que tenga poca relevancia para la vida cotidiana en países desarrollados o subdesarrollados. Afirma este estudio que esto puede ser una fuente de alienación de los estudiantes para el aprendizaje de dicha asignatura. Lo que crea la necesidad de que los objetos de estudio y los procesos del Álgebra sean significativos para los estudiantes y los docentes, que les permitan verificar que va más allá de una simple manipulación simbólica.

Esta problemática del Álgebra se ha visto reflejada en la educación venezolana, quien a lo largo de los años ha mostrado deficiencia en el proceso de enseñanza y aprendizaje de la Matemática, la investigación llevada a cabo por González, Luque y Ríos (2006) sirve como soporte a esta afirmación, al mencionar los resultados arrojados desde 1997 hasta el 2001 por la 
prueba de aptitud académica que realizaba la Oficina de Planificación del Sector Universitario (OPSU) y que medía el razonamiento verbal y la habilidad numérica de los estudiantes que aspiraban ingresar a la educación superior, muestran que en relación con la habilidad numérica el Estado donde mejor nivel presentaron los estudiantes fue el Estado Miranda con un $33 \%$ de los estudiantes que dominan los conocimientos matemáticos que deberían saber, mientras que en otros Estados los porcentajes llegan a ser tan bajos como 5\% y $3 \%$.

De igual manera, Cadenas (2007) señala, los estudiantes que ingresan a la Educación Diversificada, Profesional y Superior no dominan los conocimientos matemáticos básicos en especial en los tópicos algebraicos y estrategias generales para resolver ejercicios y para dar respuesta a interrogantes de un nivel muy elemental, como por ejemplo: operaciones con fracciones, potencia, productos notables, polinomios, ecuaciones de grado $\mathrm{n}$, funciones, conjuntos numéricos, entre otros; la cual dificulta, de esta manera, el estudio de los programas de Matemática en estos niveles educativos.

Una respuesta para este problema, es que por ser el Álgebra aquella parte de la Matemática que generaliza las leyes de la Aritmética y por estar cargada de símbolos que pueden representar cualquier cantidad, es un potencial escenario para que los estudiantes memoricen y resuelven problemas sin tener, en muchas oportunidades, un verdadero conocimiento sobre los que están haciendo; que los conduce a cometer errores y que incide de manera negativa en su formación y en su rendimiento académico.
Lo anteriormente planteado muestra que existe una gran distancia entre lo que se espera de la Educación Matemática y lo que realmente ocurre en las aulas. En el Unidad Educativa "El Molino", según los docentes que laboran allí, la enseñanza de la Matemática se realiza sin tomar en consideración los organizadores curriculares de Rico (1997) pues no tienen conocimiento de ellos, de modo que ésta, se sigue haciendo de manera formal y abstracta, desarrollada con actividades ajenas al estudiante, sin vinculación con otra área de aprendizaje, ni con su mundo físico y social, que no los ayudan a visualizar la utilidad y la pertinencia que tiene en su vida cotidiana y sin significados aparentes que los ayuden a apropiarse de los conceptos matemáticos, ni lo motivan a su estudio.

Esta institución está ubicada en el Municipio Libertador, Parroquia Tocuyito del Estado Carabobo, de acuerdo a los docentes que laborar allí, la formación de los estudiantes en Álgebra se basa en la resolución de problemas escolarizados, la ejercitación y manejo de algoritmos, con excesiva formalidad y un alto grado de abstracción, constituidas por axiomas, definiciones y teoremas aislados unos de otros y con poca aplicabilidad en la realidad del estudiante.

Las entrevistas realizadas a los estudiantes demuestran que los problemas que se les plantean en clase de Matemática, están preparados para que den un resultado único, y para que sigan un patrón o modelo idéntico al resuelto por el docente, que en muchas oportunidades, no requiere de un mayor esfuerzo por parte de ellos y que en casi la totalidad de los casos están descontextualizados de su realidad social, y 
de sus intereses lo que ha conducido a inadecuado aprendizaje de esta área.

Por su parte, el aprendizaje del Álgebra genera a los estudiantes dificultades que tienen distintas naturalezas, y al presentarla como un montón de técnicas aisladas y como procedimientos formales y mecánicos que deben aplicarse para resolver un problema, sin hacer énfasis en la vinculación con el mundo real y sin tomar en cuenta diferentes representaciones que ayuden a comprender el concepto matemático al que se está haciendo referencia, se está diseñando un escenario propicio para la formación de obstáculos que se manifiestan, como lo plantea Socas (1997), en forma de errores, los cuales son más que la formación en los estudiantes de esquemas cognitivos inadecuados (Socas, 1997). Por lo que la enseñanza del Álgebra en las instituciones debe buscar el tratamiento de los errores y procurar su eliminación.

En la búsqueda de mejorar la Educación Matemática, se han realizado numerosas investigaciones, algunas de ellas Villa, Bustamante, Berrio, Osorio y Ocampo (2008); Aravena, Caamaño y Giménez (2008); Biembengut y Hein (1999) señalan que es necesario ofrecerles a los estudiantes situaciones concretas que les permitan organizar información, describir relaciones matemáticas, enfrentar problemas con soluciones múltiples, entender la aplicabilidad de los conceptos y procesos matemáticos entre otros.

Otras investigaciones como las de Castro y Castro (1997) y Rico (2009) señalan que en toda actividad matemática deben estar presentes el uso de las representaciones o sistemas de representaciones por parte de los docentes y de los estudiantes, ya que éstas sirven para comunicar las ideas matemáticas e intervienen en la construcción de nuevos conocimientos, razón por la cual los estudiantes deben manejar el mayor número de representaciones posibles sobre un objeto matemático para facilitar su comprensión del mismo. Por lo tanto, las representaciones constituyen una actividad inseparable en los trabajos de Matemática desarrollados en las aulas.

Las respuestas de los estudiantes al instrumento presentado, deja de manifiesto que la enseñanza recibida no está haciendo suficiente énfasis en los diferentes sistemas de representación, ya que existe una estrecha relación entre sus respuestas y lo que se le enseña, los estudiantes en su mayoría trabajaron dos sistemas de representación y les costó hacer el traslado de uno a otro.

Esta realidad observada en el Unidad Educativa "El Molino" permite afirmar que los estudiantes de primer año no se apropian de los conceptos matemáticos, específicamente de los relacionados con el Álgebra, ni conciben la utilidad de la Matemática en sus vidas cotidianas, lo que los conduce a un aprendizaje aparente, memorístico y parcializado de la misma.

Debido a esta problemática, el interés de esta investigación fue estudiar los procesos seguidos por los estudiantes cuando resuelven problemas de matemáticos. En este sentido, este trabajo pretendió dar respuesta a la siguiente interrogante: ¿Qué tipo de representaciones utilizan los estudiantes de 1er. año de Educación básica, cuando resuelven problemas del mundo físico y social?, la cual se da respuesta al cumplir con el siguiente propósito: Determinar las representaciones utilizadas 
por los estudiantes de primer año cuando resuelven problemas matemáticos del mundo físico y social de la Unidad Educativa "El Molino"

Actualmente se busca la formación integral del educando, que los conocimientos que adquiere el estudiante sean útiles y aplicables en el mundo en el que viven, es por ello que se habla de un aprendizaje contextualizado que vaya en sintonía con sus necesidades y que le ayude a vivir en sociedad. Por tal motivo, la Enseñanza de la Matemática presenta en estos momentos un gran desafío; necesita ser concebida como un área que tiene pertinencia para los estudiantes y hay que hacerlo de manera que sean los propios estudiantes quienes puedan tomar conciencia de su la relevancia.

Esta necesidad de ampliar la visión de los estudiantes acerca de la aplicabilidad de la Matemática en sus vidas diarias, dio origen a la presente investigación, la cual tomó en cuenta los tópicos del Álgebra debido a la capacidad que tiene para formar habilidades que promueven el pensamiento formal, la capacidad de generalización y de abstracción; además de que puede ser considerada como un pilar fundamental en el aprendizaje de la Matemática, ya que según Katz (2007) el Álgebra elemental, que es enseñada en la escuela básica y secundaria, es la clave para el éxito de los estudiantes en toda la Matemática. Así mismo se consideró parte importante de la presente investigación, rescatar la motivación histórica para el estudio del Álgebra, que ha sido la necesidad de resolver problemas del mundo real, que le permitan a nuestros estudiantes aplicar el razonamiento crítico en su vida cotidiana (Katz, 2007).
De igual modo esta investigación considera tres de los organizadores curriculares desarrollados por Rico (1997), los cuales son necesarios para que los estudiantes integren con sentido los nuevos conocimientos y se apropien de ellos. Se considera relevante las representaciones debido a su importancia en la comprensión y comunicación del conocimiento matemático, por lo que se considera importante conocer qué tipo de representaciones utilizan los estudiantes para saber cómo es la información que poseen sobre un determinado concepto matemático, y los errores ya que estos forman parte en la adquisición del conocimiento y pueden ser una fuente inagotable de éste, si se tratan de manera adecuada y no como medio para cercenar la capacidad del estudiante, su importancia en el aprendizaje y la enseñanza de la Matemática lo hace pertenecer a los organizadores del currículo.

Esta investigación permite obtener información acerca de los errores más comunes que cometen los estudiantes de primer año cuando resuelven problemas del mundo físico y social, y la frecuencia con que estos se presentan. Siendo una información valiosa para el desarrollo del proceso de enseñanza y aprendizaje de la Matemática desde dos de sus actores, los estudiantes y los docentes. En primer lugar, los estudiantes de la Unidad Educativa "El Molino" presentan grandes dificultades en la adquisición del conocimiento matemático, específicamente en los tópicos relacionados con el Álgebra, que se reflejan en el bajo rendimiento académico; esta problemática sirvió como elemento motivador en esta investigación, y se espera que la misma sirva como un antecedente 
para las investigaciones posteriores que estén relacionadas con los estudiantes de esta institución educativa u otra similar, con el aprendizaje del Álgebra y con la resolución de problemas del mundo real.

Los resultados de esta investigación ayudan a conocer las competencias que tienen los estudiantes de esta institución para resolver problemas vinculados con su cotidianidad y así mismo destacar la importancia que tiene para el estudio del Álgebra la resolución de problemas vinculados con la vida cotidiana. Todo esto, en la búsqueda de mejorar sustancialmente el rendimiento académico de los estudiantes, y de lograr un aprendizaje significativo basado en la igualdad de oportunidades y que les permita comprender su entorno y hasta cambiarlo si es necesario.

Un antecedente relevante para este estudio fue el realizado por Castro y Castro (1997), el cual describe como aquellas notaciones simbólicas o graficas que son específicas para cada noción, mediante las que se expresan los conceptos y procedimientos matemáticos y sus características y propiedades más relevantes.

Por su parte, Rico (2009) las define como todas aquellas herramientas, signos $\mathrm{y}$ gráficos, que hacen presentes los conceptos $\mathrm{y}$ procedimientos matemáticos $\mathrm{y}$ con las cuales los sujetos abordan e interactúan con el conocimiento matemático. Debido a su importancia en la construcción de los conceptos matemáticos y el auge que ha tenido en los últimos años como objeto de investigación, el mismo Rico (2009) consideró necesario delimitar el significado de esta noción señalando que el concepto de representación hace notorio una dualidad representante- representado y que la representación sustituye algo que existe y que es distinto. La investigación de Rico permite clarificar que no se deben confundir los objetos matemáticos con sus representaciones, ya que una cosa son las herramientas, (signos, símbolos o gráficos) con las que las personas abordan el conocimiento matemático $\mathrm{y}$ otra el concepto y los procedimientos matemáticos en sí, es decir, tanto las representaciones como el "algo" que representan están estrechamente relacionados, pero tienen funciones separadas. Rico (2009) afirma, “...Es peligroso que la representaciones pretendan pasar por la presencia, el signo por la cosa misma" (p.6).

Los aspectos teóricos están orientados hacia, los sistemas de representación que utilizan los estudiantes de primer año al momento de hacer la construcción del modelo matemático dentro un proceso de modelización matemática. Las representaciones son por lo tanto un conjunto de reglas, símbolos, signos que van a expresar algunos aspectos de: conceptos, procedimientos y propiedades matemáticas y que ayudan a comprender dichos conceptos, al mismo tiempo que muestran la información que poseen las personas sobre las nociones matemáticas.

Referente a los tipos de representaciones Castro y Castro (1997) sostienen que en la adquisición y elaboración de nuevos conceptos intervienen dos tipos de representaciones que se encuentran estrechamente vinculadas, las representaciones internas: que son las que se utilizan para razonar y pensar sobre ideas matemáticas; y las representaciones externas que se utilizan para comunicar esas ideas. 
Estos tipos de representaciones también podrían ser llamadas representaciones observables y representaciones no observables, ya que las primeras solo están en la mente del sujeto y las segundas se ponen de manifiesto o se muestran en la realización del trabajo matemático. Para Castro (1997) las representaciones internas son necesarias para pensar y razonar ideas matemáticas y para que la mente tenga la posibilidad de operar con ellas, mientras que las representaciones externas son necesarias para comunicar estas ideas $\mathrm{y}$ hacerlas accesibles a los demás. De igual manera el autor antes mencionado señala la relación que existe entre estas dos tipos de representaciones y en la doble función que cumplen las representaciones externas:

1. Actúan como estímulo de los sentidos en los procesos de construcción de nuevas estructuras mentales

2. Como expresión de los conceptos e ideas que poseen los sujetos que los utilizan.

Como ya se ha mencionado las representaciones externas expresan las ideas que los personas tienen de un objeto o concepto matemático, razón por la cual en esta investigación sólo se tomará en cuenta este tipo de representación; dentro de las representaciones externas existen una gran variedad de representaciones que se pueden utilizar para describir un objeto matemático, tantas que no se halla una clasifica específica de todas estas, sin embargo las han divido en dos grandes familias, consideradas por Castro y Castro (1997) como las representaciones o sistemas de representaciones simbólicas que se basan en signos alfanuméricos estructurados. Y las representaciones o sistemas de representación gráfica que son combinaciones de figuras o iconos estructurados.

Debido a la importancia que tienen los sistemas de representación en el estudio de la matemática diversos investigadores han centrado el estudio de las representaciones de los conceptos matemáticos escolares en diversos sistemas simbólicos y gráficos así como en las relaciones entre ellos, al respecto Castro y Castro (1997) señala los trabajos de Janvier, Kaput, Goldin y Duval, quienes han encontrado para los contenidos matemáticos de educación secundaria en primer lugar los sistemas numéricos y los algebraicos y en segundo lugar los sistemas basados en las medidas, la recta real, el plano cartesiano, entre otros.

Estas estructuras simbólicas y gráficas son las que ayudan a los estudiantes a comprender los conceptos matemáticos, por lo tanto mientras más sistemas de representación domine el estudiante mayor será su comprensión del concepto. En relación con esto, Castro y Castro (1997) afirman que una persona domina un concepto matemático cuando:

1. Conoce sus principales representaciones y el significado de cada una de ellas.

2. Opera con las reglas internas de cada sistema.

3. Convierte una representación en otra, es decir, cuando es capaz de pasar de una representación a otra.

4. Puede detectar cuál sistema es más ventajoso para trabajar con determinadas propiedades. 
Se entenderá como Sistemas de representaciones al conjunto de símbolos, gráficos y reglas que permiten representar una estructura matemática y que han de responder a su carácter sistémico (Castro y Castro, 1997). No obstante, ningún sistema de representación por más amplio que sea puede describir en su totalidad algún concepto matemático, ellos muestran información parcial de alguna característica del concepto estudiado, tal como lo plantean Castro (1997), Castro y Castro (1997) y Rico (2009) al afirmar que los conceptos matemáticos vienen expresados a través de varios sistemas de representación específicos, y que un mismo objeto puede ser enseñado mediante representaciones diferentes; ya que ningún sistema de representación agota por si sólo un concepto, ni las complejas relaciones que estos encierran, si no que cada uno de ellos transmite $o$ muestra una parte específica del mismo; una propiedad, una característica diferente, por lo que se considera pertinente que en el aprendizaje de los objetos matemáticos se utilicen varios sistemas de representación, que al trabajarlos en conjunto le permitan al estudiante comprender y asignarle significados a los distintos aspectos de un concepto matemático.

\section{MÉTODO}

El estudio se enmarcó dentro del paradigma interpretativo con un enfoque cualitativo porque pretendió determinar las representaciones utilizadas por los estudiantes de primer año cuando resuelven problemas matemáticos del mundo físico y social. Para ello, se recurrió al estudio de caso, el cual según Stake (1999) “es el estudio de la particularidad de un caso singular, para llegar a comprender su actividad en circunstancias importantes" (p. 11), siendo el caso en esta investigación un grupo de estudiantes del primer año de Educación Media.

El foco de interés se centró en las producciones realizadas por el grupo seleccionado para a partir de las representaciones utilizadas por los estudiantes en la resolución de problemas matemáticos del mundo físico y social. Las fases ejecutadas fueron:

1. Revisión de la Literatura: En esta primera fase se llevó a cabo la recopilación de la información pertinente y la lectura de artículos e investigaciones recientes, provenientes de revistas, actas de congresos, libros y trabajos de Maestría culminados con la finalidad de aumentar la comprensión del problema, ubicar el problema en un contexto nacional e internacional, reconocer los autores claves y apropiarse de las teorías que sustentan la presente investigación.

2. Diseño: En esta fase se diseñó y validó los instrumentos, que fueron aplicados a los participantes en el estudio. El diseño de los instrumentos se sometió a discusiones en el grupo de trabajo de la Línea de Investigación Pensamiento Numérico y Algebraico (Ortiz, 2004) y la validación se efectuó a través de la valoración de expertos. Para el diseño de los instrumentos, previamente se realizó una reunión o primer contacto con los estudiantes sujetos de investigación, a fin de conocer sus inquietudes y aquellos aspectos o fenómenos de su realidad que 
son relevantes para ellos. El propósito de esta reunión fue captar aspectos significativos de sus contextos para a partir de allí proceder a la formulación de las situaciones problemas que se les aplicó posteriormente. Así mismo se consideraron las técnicas que permitirán recoger la información necesaria en correspondencia con el tipo de investigación.

\section{Aplicación: Correspondió en esta} fase la aplicación del cuestionario contentivo de seis (6) problemas del mundo físico y social, es decir, del mundo real de los estudiantes. Se llevó a cabo en dos sesiones de trabajo en las que cada grupo de participantes, luego de recibir los instrumentos correspondientes, resolvió tres problemas por sesión. Éstas se desarrollaron siguiendo tres momentos clave: Introducción, Resolución y Discusión de los problemas propuestos. En cada sesión, se contó con la presencia de un investigador invitado, como observador, para facilitar el registro de la información. Así mismo, en esta fase se recogió la información pertinente para la investigación producto de las actividades desarrolladas por los participantes en el estudio, así como de las notas de campo recogidas por los investigadores.

\section{Análisis: Aquí se procedió al} análisis de la información recogida en las producciones de los sujetos participantes y de los comentarios aportados por las notas de campo, derivación de la observación realizada y de los hallazgos encontrados. Como resultado del análisis se generaron las conclusiones pertinentes sobre las representaciones utilizadas por los estudiantes de primer año cuando resuelven problemas matemáticos del mundo físico y social.

Por otra parte, inicialmente el estudio estaba contemplado para veinte (20) estudiantes voluntarios del 1er año de Educación Media de la Unidad Educativa“El Molino" con edades comprendidas entre 12 y 14 años que asistían con regularidad a sus clases de Matemática. Condiciones indispensables como criterio para la participación como informantes de la investigación. Sin embargo, en la primera sesión de trabajo asistieron treinta (30) estudiantes, diez (10) más de lo que se tenía planteado; por lo que tomando en cuenta su motivación para participar en la actividad, se les permitió formar parte de la misma.

La técnica empleada para la recolección de la información fue la observación participante; como miembro de la institución Unidad Educativa "El Molino", la investigadora efectuó la observación participante haciendo uso del cuaderno de notas de campo. El foco de atención de la observación fueron las diferentes fases del proceso. También se contó con otros observadores participantes, profesores de la institución del área de matemática, quienes funcionaron como apoyo para registrar todas las incidencias de las dos sesiones de trabajo, tomando notas de las preguntas y observaciones de los grupos de estudiantes. Además de la entrevista en profundidad; esta técnica se empleó, una vez concluidas las dos sesiones de trabajo, con el propósito de aclarar algunos 
aspectos de interés relativos a las impresiones de los estudiantes sobre la actividad realizada.

Los instrumentos empleados fueron: el cuestionario de problemas: contentivo de seis (6) problemas del mundo físico y social, derivados de la realidad de los estudiantes, y el guion de la Entrevista: o plan detallado para llevar a cabo la entrevista, pues según Stake (1999) es muy fácil no realizar las preguntas adecuadas y muy difícil dirigir a los informantes al tema de interés. El diseño de la entrevista estuvo orientado a verificar individualmente las competencias en las representaciones utilizadas por los estudiantes de primer año cuando resuelven problemas matemáticos del mundo físico y social; de modo que las interrogantes de la entrevistas estaban relacionadas con los indicadores de cada competencia.

\section{RESULTADOS Y DISCUSIÓN}

Los sistemas de representaciones utilizados por los grupos fueron analizados tomando en cuenta a Castro (1997), Castro y Castro (1997) y Rico (2009). Se obtiene como primer resultado, las representaciones que fueron utilizadas en los modelos matemáticos realizados por los estudiantes predomina el lenguaje natural, tal como lo plantea Castro y Castro (1997) al afirmar que el sistema de representación verbal es inseparable del proceso de enseñanza y aprendizaje de la Matemática.

En segundo lugar el sistema más utilizado fue el numérico y sólo un grupo logró hacer uso del sisma algebraico, a pesar de que en este nivel los estudiantes ya han trabajado con expresiones algebraicas.

Las respuestas dadas por los estudiantes, de cierta manera, se relacionan con la enseñanza que han recibido, lo que hace suponer que los docentes podrían no estar haciendo énfasis en el uso de las diferentes representaciones al tratar un concepto matemático, por lo que se sugiere que en las actividades de matemática se trabaje con diferentes representaciones y con la conversión de una representación a otra para asegurar un aprendizaje significativo de un concepto matemático, tal como lo plantea, Castro y Castro (1997) y Rico (2009).

Las entrevistas a los estudiantes arrojaron que sólo dos (2) de los veintisiete (27) estudiantes entrevistados son capaces de utilizar un sistema de representación algebraico simple como lo es una ecuación lineal y con mucha dificultad. Esto pone de manifiesto la necesidad de incorporar la resolución de problemas no escolarizados, no preparados para arrojar una solución única, que permitan desarrollar en el estudiante un pensamiento crítico y que promocione el trabajo de construcción de modelos algebraicos.

\section{CONCLUSIONES}

Es necesario abordar los contenidos matemáticos a través de la resolución de problemas reales que preparen a los estudiantes para conocer $y$, si es necesario, modificar su entorno. Además formar a los estudiantes para el estudio del álgebra, pues el sistema más utilizado por los estudiantes fue el verbal $y$ numérico. No obstante el sistema de 
representación algebraico estuvo prácticamente ausente.

La resolución de problemas se debería centrar en la Modelización Matemática como herramienta que vincula la matemática con la realidad del educando y que se establezca como prioridad el uso de diferentes sistemas de representación y el traslado de un sistema a otro; para garantizarle al estudiante una mayor comprensión de todas las propiedades y características de un concepto matemático, debido a que no se está haciendo énfasis en el uso de los diferentes sistemas de representaciones durante el proceso de enseñanza y aprendizaje dentro del aula de clase.

Continuar investigando sobre el potencial de las representaciones utilizadas por los estudiantes al momento que resuelven problemas matemáticos del mundo físico y social en la Educación Media, a fin de seguir sentando bases para mejorar el proceso de enseñanza y aprendizaje de la matemática en este nivel educativo.

\section{REFERENCIAS}

Aravena, M., Caamaño, C. y Giménez, J. (2008). Modelos Matemáticos a través de Proyectos. Revista Latinoamericana de Investigación en Matemática Educativa. 11. 49-92. Recuperado de http://redalyc.uaemex.mx/src/inicio/ ArtPdfRed.jsp?iCve=33511103\&iCveNu $\mathrm{m}=7961$

Biembengut, M. y Hein, N. (1999). Modelación Matemática: Estrategia para enseñar y aprender matemáticas. Educación Matemática. 11(1), 119-134

Cadenas, R. (2007). Carencias, Dificultades y Errores en los Conocimientos Matemáticos en Alumnos del Primer
Semestre de la Escuela de Educación de la Universidad de los Andes. Revista Orbis/ Ciencias Humanas. 2 (6), 68-84. Recuperado de http://www.revistaorbis.org.ve/6/6art 4.pdf

Castro, E. (1997). Configuraciones puntuales. Sistema de representación idóneo para las sucesiones de números naturales. CIBEM, 1 (20), 1-20. Recuperado de http://www.educ.fc.ul.pt/docentes/jp onte/DA/DATEXTOS/Castro(CIBEM).pdf

Castro, E. y Castro E. (1997). Representaciones y Modelización, en L, Rico (Coord). La Educación Matemática en la Enseñanza Secundaria. (cap. 4, pp 95-122,) Barcelona: Horsori

González, M.; Luque, R. y Ríos, (2006). Habilidades y destrezas matemáticas en alumnos que ingresan a la Escuela de Educación. ED. 3(1), 30-48. Recuperado de http://www.serbi.luz.edu.ve/scielo.ph p?script=sci_arttext\&pid=S131540792006004000003\&lng=es\&nrm=iso> Jaime, A., y Gutiérrez, A. (1990). Una propuesta de fundamentación para la educación de la Geometría: El modelo de Van Hiele. Teoría y práctica en educación matemática, 303-376

Katz, V. J. (2007). Stages in the history of Algebra with implications for teaching. Educational Studies in Mathematics 66(2), 185-201

Luengo González, R., Blanco Nieto, L., Mendoza García, M., Sánchez Pesquero, C., Márquez Zurita, L. y Casas García, L.M. (1997). Proporcionalidad Geométrica y Semejanza. Madrid: Editorial Síntesis, S.A

PISA. (2009). Programa para la Evaluación Internacional de los Alumnos OCDE. Informe español. Ministerio de Educación Gobiernos de España. Recuperado de 
www.educacion.es/cesces/actualidad/p isa-2009-informe-espanol.pdf

Ortiz, J. (2004). Pensamiento Numérico y Algebraico. Paradigma, 25(1), 225-239

Rico, L. (1997). Los organizadores del currículo de matemáticas. En Rico, L. Dir., Castro, E., Castro, E., Coriat, M., Marín, A., Puig, L., Sierra, M., Socas, M.M. (Coords). La educación matemática en la enseñanza secundaria. Ice - Horsori, pp. 39 - 59

Rico, L. (2009). Sobre las nociones de Representación y Comprensión de la Investigación en Educación. PNA 4(1), 1-14. Recuperado de http://www.pna.es/Numeros/pdf/Rico 2009Sobre.pdf

Socas, M. (1997). Dificultades, Obstáculos y errores en el aprendizaje de la Matemática en la Educación Secundaria. En L. Rico (Coord.) La Educación Matemática en la Enseñanza Secundaria (cap. 5, pp.125-154). Barcelona: Horsori

Socas, M.; Camacho, M. y Hernández, J. (1998). Análisis Didáctico del Lenguaje algebraico en la Enseñanza Secundaria. Revista Interuniversitaria de Formación del Profesorado. № 2, 73-86. Recuperado de dialnet.unirioja.es/servlet/fichero_artí culo? codigo $=117980$ \&orden $=86395$

Stacey, K y Chick H. (2004).Solving the Problema with Algebra. En K. Stacey, H. Chick y M. Kendal (Coords.). The Future of the Teaching and Learning of Algebra. (The 12th ICMI Study, cap. 1.1, pp. 1-18).New York: Kluwer Adademic Publishers

Stake, R. (1999). Investigación con Estudio de Casos (Segunda edición). Madrid: Morata

Villa, J., Bustamante, C., Berrio, M., Osorio, A. y Ocampo, D. (2008). El Proceso de Modelación Matemática en las Aulas Escolares. Recuperado de http://74.125.47.132/search?q=cache:E bhlWyAP5bsJ:ayura.udea.edu.co/mood le/file.php/56/Cursos_en_Asocolme_2 008.pdf+modelizaci\%C3\%B3n+matem\% C3\%A1tica+en+educaci\%C3\%B3n+b\%C3 $\% A 1$ sica\&cd=14\&hl=es\&ct=clnk\&gl=ve 